\title{
LEARNING ANALYTICS AND ITS DATA SOURCES: WHY WE NEED TO FOSTER ALL OF THEM
}

\author{
Dirk Tempelaar \\ Maastricht University School of Business and Economics \\ Tongersestraat 53, 6211 LM, Maastricht, Netherlands
}

\begin{abstract}
The search for rigor in learning analytics applications has placed survey data in the suspect's corner, favoring more objective trace data. A potential lack of objectivity in survey data is the existence of response styles, the tendency of respondents to answer survey items in a particular biased manner, such as yeah saying or always disagreeing. Making use of multiple survey instruments that exhibit similar types of response styles, our empirical study identifies response style bias by estimating the aggregate level of a set of response styles, amongst them the Acquiescence Response Style and the Dis-Acquiescence Response Style. We next demonstrate that trace variables are indeed bias-free in that their estimated response style components are small in size, accounting for minimal explained variation. Remarkably, course performance data is not bias-free, implying that predictive modelling for learning analytics purposes will, in general, profit from the inclusion of these bias components or apply survey data containing such response style bias to increase predictive power.
\end{abstract}

\section{KEYWORDS}

Learning Analytics, Trace Data, Survey Data, Course Performance Data, Response Styles

\section{INTRODUCTION}

What makes data rigorous for Learning Analytics (LA)? That data-related question is one of several in the quest for rigour in LA, opened by the editors in chief of the Journal of Learning Analytics: Knight, Wise, and Ochoa (2019). First directions in that quest are enclosed in the eight SOLAR webinar titled 'What Do We Mean by Rigour in Learning Analytics?' (SOLAR, 2020). Those directions pertain to several facets of LA research: beyond data, data types, data sources and data collection, also research methods and analysis procedures are concerned. The data orientation for LA as provided in the SOLAR webinar is voiced by Philip Winne, as part of the perspective of rigour in statistical approaches in LA research. In his contribution, Winne focuses on types of data being good enough for LA, with a clear suggestion for its direction: "What is a goal for LA? LA might strive to accurately and responsibly predict achievement and motivation from event data so that we can dispense with, get rid of, tests and surveys. Can I tell whether you have learned by just observing what you do as a learner and how you feel about yourself as a learner by just looking at what you do?" (SOLAR, 2020).

Banning all data from LA other than computer-logged event data indeed satisfies a crucial aspect of rigour: its restrictiveness. But does it do justice to the goal of accurately and responsibly predicting achievement and motivation? A wealth of empirical LA research applies other data types than event data in successfully predicting learning-related phenomena (for an overview of data types that contribute to LA-based predictions (see e.g., Ifenthaler and Yau, 2020; Dawson et al., 2019). There is even a branch within LA, called Dispositional LA, that entirely builds on the potential of combining event data with survey-based disposition data (Buckingham Shum and Deakin Crick, 2012). It is unclear what criteria would suggest that each and any research within this research tradition lacks rigour.

If anything, a candidate for such a criterion is subjectivity: computer-logged event data are objective, self-report survey data are subjective. For a moment renouncing that all data is subjective in the sense of being fabricated and thus containing bias (SOLAR, 2020; Winne, 2020), the popular view is that subjective data is biased, in contrast to objective data, and therefore objective data is to be preferred. A view easy to unravel: also objective data is subject to error. A simple example is connect-time in a digital learning system: a very 
objective measure, but as soon as it is applied as a measure of time-on-task, it contains the bias of including idle time not used to work on the task at hand. However, in this contribution, we aim to follow a different rationale: that of profiting from the fact of a bias component within subjective data. The rationale has two distinct features. First, it is often possible to operationalize the bias component and by doing so, correct the subjective measure for the bias. Second: the bias component itself may have predictive power towards crucial response variables in our prediction model, in which case predictive power increases by including the bias estimate together with the corrected measure in our prediction model, or include the uncorrected, biased predictor variable only, when model parsimony of prediction models matters.

Our contribution is directed at the estimation and subsequent correction for one specific bias in self-reported survey data: the presence of response styles in survey data. Response styles represent characteristic patterns in the answers to surveys that act as personality traits, such as the tendency to yeah saying or to give extreme responses. Response styles in survey data are extensively analysed in different academic disciplines, like marketing (Baumgartner and Steenkamp, 2001; Weijters, Cabooter, and Schillewaert, 2010), cultural studies (Cheung and Rensvold, 2000) and psychometrics (Bolt, Lu, and Kim, 2014; Henninger and Meiser, 2020a, 2020b). Different solutions to assess the presence of response styles type of bias have been proposed, like the introduction of neutral, bias-free items that serve as standards for the biased items, the anchoring vignettes (Bolt et al., 2014) or the use of item-response theory models (Henninger and Meiser, 2020a). This contribution builds on previous research of the authors (Tempelaar, Rienties, and Nguyen, 2020), where we use an aggregated measure of response styles as to identify the 'bias component' in different types of data. Estimating such an aggregate response style score is possible by the availability of data from multiple survey instruments and the relative invariance of these instrument-specific response styles (Tempelaar et al., 2020). In this contribution, we focus on the role of the three oldest response styles described in the literature: the Acquiescence Response Style, the systematic tendency to confirm item statements, the Dis-Acquiescence Response Style, the tendency to disagree with item statements, and the MidPoint Response Style, the tendency to respond neutrally. The role of these response styles in building predictive models is the main focus of the current study.

\section{METHODS}

\subsection{Response Styles}

Response styles refer to typical patterns in responses to Likert response scales questionnaire items (Baumgarten and Steenkamp, 2001; Weijters et al., 2010). Response styles are induced by the tendency of respondents to respond in a similar way to items, independent of the content of the item, such as yeah saying, or seeking for extreme responses. In the literature, eleven common types of response styles are distinguished:

- Acquiescence Response Style, ARS: the tendency to respond positively

- Dis-Acquiescence Response Style, DARS: the tendency to respond negatively

- $\quad$ Net-Acquiescence, NARS: ARS-DARS

- MidPoint Response Style, MRS: the tendency to respond neutrally

- Non-Contingent Response, NCR: the tendency to respond at random

- Extreme Response Scale, ERS: the tendency to respond extremely

- Extreme Response Scale, ERSpos and ERSneg: the tendency to respond extremely positively or extremely negatively

- Response range, RR: the difference between the maximum and minimum response

- $\quad$ Mild Response Style, MLRS: the tendency to provide a mild response.

Longitudinal research into the stability of response styles concludes that response styles function as relatively stable, individual characteristics that can be included as control variables in the analysis of questionnaire data (Weijters et al., 2010). Empirical studies tend to focus on the role of ERS only; for that reason, our previous study (Tempelaar et al., 2020) stayed in that tradition. Response styles constitute a collinear set of observations, by definition: for example, mild responses are the complement of extreme responses. 


\subsection{Dispositional Learning Analytics}

Dispositional learning analytics proposes a learning analytics infrastructure that combines learning data, generated in learning activities through the traces of technology-enhanced learning systems, with learner data, such as student dispositions, values, and attitudes measured through self-report questionnaires (Buckingham Shum and Deakin Crick, 2012). In our dispositional learning analytic research (Tempelaar et al., 2020), we operationalised dispositions with the help of instruments developed in the context of contemporary social-cognitive educational research, as to make the connection with educational theory as strong as possible. Another motivation to select these instruments is that they are closely related to educational interventions. These instruments include:

- The expectancy-value framework of learning behaviour, encompassing affective, behavioural, and cognitive facets;

- The motivation and engagement framework of learning cognitions and behaviours that distinguishes learning cognitions and learning behaviours of adaptive and maladaptive types;

- Aspects of a student approach to learning (SAL) framework: cognitive processing strategies and metacognitive regulation strategies;

- The control-value theory of achievement emotions, both about learning emotions of activity and epistemic types, at the affective pole of the spectrum;

- Goal setting behaviour in the approach and avoidance dimensions;

- Academic motivations that distinguish intrinsically versus extrinsically motivated learning.

\subsection{Context of the Study}

This study took place in a large-scale introductory course in mathematics and statistics for first-year students in a business and economics course in the Netherlands. The education system can best be described as 'blended' or 'hybrid'. The face-to-face component is Problem-Based Learning (PBL), in small groups, coached by a tutor. The online component of the mix was two e-tutorials to learn and practice mathematics and statistics. Since most of the learning occurs in outside class self-study through the e-tutorials or other teaching materials, the teaching time is used to discuss advanced problem-solving, as in flipped-classroom design.

The subject of this study is the entire cohort of students in $2018 / 2019$, i.e. all students who registered for the course and responded to the learning dispositions instruments: a total of about 1100 students. There was a great diversity in nationality in the student population: only $22 \%$ were educated in the Dutch secondary school system, in total of 57 nationalities were present. International education systems differ widely, especially in mathematics and statistics. Therefore, it is crucial that this current introductory module is flexible and allows for individual learning pathways, which is why we opt for a blended design with a lot of learning feedback from the students through the application of dispositional learning analytics.

In this research, we combine data from different types: course performance, Learning Management System (LMS) and e-tutorial trace variables, and learning dispositions variables measured with self-report questionnaires, in line with Winne's taxonomy of data sources (Winne, 2020).

The self-report questionnaires applied in this research are fully described in our (open-access) previous study (Tempelaar et al., 2020); for reasons of space, we will provide only a short overview here.

- Learning activity emotions from the AEQ instrument: Enjoyment, Anxiety, Boredom, Hopelessness, and their direct antecedent, Academic Control.

- Epistemic emotions, EES instrument: Surprise, Curiosity, Confusion, Anxiety, Frustration, Enjoyment, and Boredom.

- Achievement goals, AGQ instrument: Task-Approach, Task-Avoidance, Self-Approach, Self-Avoidance, Other-Approach, Other Avoidance, Potential-Approach, and Potential-Avoidance Goals.

- Motivation and engagement, MES instrument: Self-belief, Value of school, Learning focus, Planning, Study management, Persistence, Academic buoyancy, Anxiety, Failure avoidance, Uncertain control, Self-sabotage, and Disengagement.

- Attitudes to learning, SATS instrument: Affect, Cognitive competence, Value, No difficulty, Interest, and Effort. 
- Approaches to learning, ILS instrument: Memorising and rehearsing, Analysing, Relating and structuring, Critical processing, Concrete processing, Self-regulation of learning processes and results, Self-regulation of learning content, External regulation of learning processes, External regulation of learning results, and Lack of regulation.

- Academic motivations, AMS instrument: Intrinsic motivation to know, to accomplish, to experience stimulation, Identified, Introjectd and External regulation, and Amotivation.

The final course performance measure, Grade, is a weighted average of the final exam score (87\%) and the quiz score $(13 \%)$. The performance in the exam has two components of equal weight: the math exam score (MathExam) and the exam score statistics (StatsExam). The same decomposition refers to the aggregated performance in the quizzes for both subjects: MathQuiz and StatsQuiz.

Trace data from technology-enhanced learning systems related the LMS BlackBoard and two e-tutorials systems for mathematics and statistics. BBClicks as the total number of clicks in BlackBoard is most predictive for performance. From the trace variables available from the two e-tutorial systems, we selected process variables representing the number of attempts to solve exercises: MathAttempts and StatsAttempts, and total time on task: MathTime and StatsTime. The math e-tutorial system then archives the feedback strategies that students use when solving an exercise, resulting in additional process variables MathHints, the total number of hints requested, and MathSolutions, the number of worked examples requested.

\subsection{Data Analysis}

All disposition questionnaires were administered with items of the Likert $1 \ldots 7$ type. A set of 11 response styles was calculated for all seven questionnaire administrations: ARS, ARSW, DARS, DARSW, MRS, NARS, NARSW, RR, NCR, ERSneg, and ERSpos. By definition, this set of response styles was strongly collinear, making a selection necessary. In this study, we opted for ARS, DARS and MRS.

After estimating aggregated ARS, DARS, and MRS levels for all students in the sample, we applied an instrumental variables approach. All variables were regressed on the ARS and DARS response styles (MRS is left out for collinearity), allowing to decompose all variables into two, orthogonal components: the part of the variable explained by the response styles and the part that is left unexplained (the residual of the regression). The beta weights describe that decomposition. To provide an example: the AEQ variable learning anxiety (LAX), when regressed on the response styles ARD and DARS, generated the following regression equation: LAX $=0.100($ ARS $)-0.537($ DARS $), \mathrm{R}^{2}=0.367$.

This procedure was applied to all variables under study, including the 'objectively' measured variables. That is, self-report constructs, trace variables of the process and product types, and course performance variables were all assigned variable-specific scores for ARS and DARS.

\section{RESULTS}

\subsection{Response Styles Descriptives}

Descriptive statistics of response styles of different instruments are provided in Table 1. There are considerable differences in these statistics between the instruments, which can be explained by the balance between adaptive or positive items in the instrument on the one side and negative or maladaptive items on the other side. For instance, the highest ARS score is for the achievement goal instrument AGQ, since all its items correspond to achievement motives that are part of most students' goal-setting behaviour. In contrast, DARS scores are high for the AEQ instrument, which counts several items relating to negative emotions.

There exists collinearity amongst the set of response styles, resulting from the overlap in their definitions. For instance, MRS is the complement of ARS and DARS, and therefore we will restrict the subsequent analysis to ARS and DARS, leaving aside MRS. 
Table 1. Descriptive statistics of response styles measures and the overall mean and median of all response styles

\begin{tabular}{lrrrrrrrrr}
\hline & Mean & Median & MES & EES & AGQ & ILS & AEQ & AMS & SATS \\
\hline ARS & 0.56 & 0.56 & 0.57 & 0.45 & 0.80 & 0.61 & 0.33 & 0.61 & 0.54 \\
DARS & 0.28 & 0.28 & 0.33 & 0.30 & 0.08 & 0.22 & 0.51 & 0.25 & 0.31 \\
MRS & 0.16 & 0.15 & 0.10 & 0.24 & 0.12 & 0.18 & 0.16 & 0.14 & 0.15 \\
NARS & 0.28 & 0.28 & 0.24 & 0.15 & 0.73 & 0.39 & -0.18 & 0.36 & 0.23 \\
RR & 5.17 & 5.14 & 5.86 & 4.34 & 3.49 & 4.84 & 5.03 & 5.68 & 6.95 \\
NCR & 1.16 & 1.15 & 0.76 & 1.69 & 1.13 & 0.98 & 1.28 & 1.07 & 1.22 \\
ERSneg & 0.15 & 0.13 & 0.26 & 0.16 & 0.04 & 0.09 & 0.34 & 0.06 & 0.20 \\
ERSpos & 0.34 & 0.34 & 0.40 & 0.19 & 0.63 & 0.28 & 0.13 & 0.42 & 0.35 \\
MRLS & 0.49 & 0.49 & 0.34 & 0.66 & 0.32 & 0.63 & 0.54 & 0.52 & 0.45 \\
\hline
\end{tabular}

\subsection{Individual Response Style Scores}

The next step after determining response style scores for each individual student in each disposition instrument was to average these scores over all seven instruments, generating aggregate ARS and DARS scores for all students. The pattern that arises is pictured in Figure 1: all 1113 students are included in the scatter. Overall, there is a clear negative relationship between ARS and DARS scores: the correlation equals $r=-.646$. Such a negative relationship is natural: the more items a student answers in the acquiescence range, the fewer items are left over to provide answers in the dis-acquiescence range or neutral. In this paper, we will not discuss individual response style scores in more detail but only use these scores as instruments to derive the response style composition of variables.

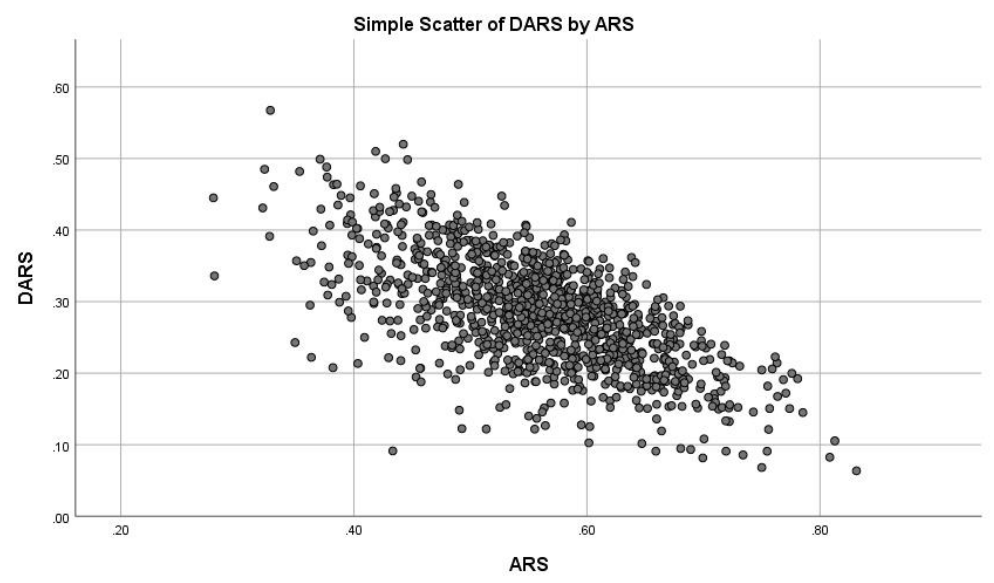

Figure 1. Scatterplot of aggregated ARS, acquiescence scores versus aggregated DARS, dis-acquiescence scores, for all 113 students

\subsection{Classification of Variables based on Response Styles}

The availability of response style measures allows new ways to categorise our data in educational studies. Rather than using the dichotomy of self-reported data versus objectively scored, we can position each variable of each data type in a two-dimensional plane of response styles: ARS and DARS. The data required for this presentation is in Table 2. It provides for all variables in the analysis the two response style components: the betas of the regression of the variable on ARS and DARs, representing the share of ARS and the share of DARS in explained variation. That explained variation is provided in the last column of Table 2 , as the $\mathrm{R}^{2}$ percentage. 
Table 2. Response style components of course performance variables, trace variables and disposition variables, together with explained variation by the ARS and DARS response styles

\begin{tabular}{|c|c|c|c|c|c|c|c|}
\hline & ARS & DARS & $\mathrm{R}^{2}$ & & ARS & DARS & $\mathrm{R}^{2}$ \\
\hline Course performance & & & & Trace variables & & & \\
\hline Grade & 0.129 & 0.405 & $11.3 \%$ & BBclicks & 0.093 & 0.017 & $0.7 \%$ \\
\hline MathExam & 0.090 & 0.345 & $8.6 \%$ & StatsTime & 0.056 & -0.080 & $1.5 \%$ \\
\hline StatsExam & 0.138 & 0.387 & $9.9 \%$ & StatsAttempts & 0.098 & -0.033 & $1.5 \%$ \\
\hline MathQz & 0.137 & 0.304 & $5.8 \%$ & MathTime & 0.096 & -0.048 & $1.8 \%$ \\
\hline StatsQz & 0.088 & 0.250 & $4.2 \%$ & MathAttempts & 0.093 & -0.115 & $3.5 \%$ \\
\hline \multicolumn{4}{|c|}{ Motivation \& Engagement Scale } & MathSolutions & 0.013 & -0.217 & $5.1 \%$ \\
\hline Self-Belief & 0.423 & 0.404 & $12.1 \%$ & MathHints & 0.024 & -0.037 & $0.3 \%$ \\
\hline Persistence & 0.422 & 0.235 & $10.5 \%$ & Learning strategies & & & \\
\hline Learning Focus & 0.517 & 0.213 & $17.0 \%$ & Relating \& structure & 0.546 & 0.230 & $19.1 \%$ \\
\hline Value School & 0.481 & 0.214 & $14.4 \%$ & Critical process. & 0.337 & 0.078 & $8.6 \%$ \\
\hline Study Managm & 0.328 & 0.099 & $7.5 \%$ & Memorise \& rehear & 0.338 & -0.040 & $13.3 \%$ \\
\hline Planning & 0.351 & 0.095 & $8.9 \%$ & Analysing & 0.486 & 0.115 & $17.8 \%$ \\
\hline Ac Buoyancy & -0.044 & 0.264 & $8.7 \%$ & Concrete proc. & 0.456 & 0.111 & $15.6 \%$ \\
\hline Disengament & -0.308 & -0.362 & $8.1 \%$ & SelfRegulatingProc & 0.466 & 0.056 & $18.7 \%$ \\
\hline Self Sabotage & -0.197 & -0.331 & $6.4 \%$ & SelfRegulatingCont & 0.291 & -0.060 & $11.1 \%$ \\
\hline Uncert Contr & -0.100 & -0.466 & $16.7 \%$ & ExternalRegulProc & 0.367 & 0.018 & $12.7 \%$ \\
\hline Failure avoid & 0.023 & -0.341 & $12.5 \%$ & ExternalRegulCont & 0.543 & 0.236 & $18.7 \%$ \\
\hline Anxiety & 0.142 & -0.408 & $26.2 \%$ & Lack of Regulation & -0.005 & -0.496 & $24.3 \%$ \\
\hline \multicolumn{4}{|l|}{ Epistemic Emotions } & \multicolumn{4}{|l|}{ Achievement Goals } \\
\hline Curious & 0.405 & 0.151 & $10.8 \%$ & Task Approach & 0.382 & 0.267 & $8.6 \%$ \\
\hline Surprised & 0.185 & -0.187 & $11.3 \%$ & Task Avoid & 0.339 & 0.127 & $7.6 \%$ \\
\hline Confused & 0.036 & -0.445 & $22.0 \%$ & Self Approach & 0.484 & 0.143 & $16.6 \%$ \\
\hline Anxious & 0.111 & -0.503 & $33.8 \%$ & Self Avoid & 0.447 & 0.096 & $15.4 \%$ \\
\hline Frustrated & -0.083 & -0.498 & $20.2 \%$ & Other Approach & 0.288 & 0.029 & $7.3 \%$ \\
\hline Excited & 0.299 & 0.164 & $5.3 \%$ & Other Avoid & 0.343 & 0.011 & $11.1 \%$ \\
\hline Bored & -0.290 & -0.381 & $8.7 \%$ & Potential Approach & 0.438 & 0.166 & $12.6 \%$ \\
\hline \multicolumn{4}{|l|}{ Activity Emotions } & Potential Avoid & 0.435 & 0.092 & $14.6 \%$ \\
\hline Learn Anxiety & 0.100 & -0.537 & $36.7 \%$ & \multicolumn{4}{|c|}{ Academic Motivation Scale } \\
\hline Learn Boredom & -0.317 & -0.435 & $11.3 \%$ & IntrinMotivKnow & 0.516 & 0.125 & $19.9 \%$ \\
\hline Learn Hopeless & -0.106 & -0.618 & $31.0 \%$ & IntrinMotivAccom & 0.528 & 0.027 & $26.1 \%$ \\
\hline Learn Enjoym & 0.381 & 0.161 & $9.2 \%$ & IntrinMotivStim & 0.358 & -0.113 & $19.4 \%$ \\
\hline Acad Control & 0.236 & 0.585 & $22.1 \%$ & ExtrinMotivIden & 0.422 & 0.137 & $12.2 \%$ \\
\hline \multicolumn{4}{|c|}{ Attitudes towards learning math \& stats } & ExtrinMotivIntro & 0.414 & -0.117 & $24.7 \%$ \\
\hline Affect & 0.078 & 0.515 & $21.9 \%$ & ExtrinMotivExt & 0.311 & 0.035 & $8.4 \%$ \\
\hline CognCompeten & 0.107 & 0.490 & $18.4 \%$ & Amotivation & -0.234 & -0.344 & $7.0 \%$ \\
\hline Value & 0.368 & 0.404 & $10.7 \%$ & & & & \\
\hline No Difficulty & -0.192 & 0.111 & $7.7 \%$ & & & & \\
\hline Interest & 0.395 & 0.175 & $9.8 \%$ & & & & \\
\hline Effort & 0.382 & 0.094 & $10.8 \%$ & & & & \\
\hline
\end{tabular}

There exist large differences in the role of the ARS and DARS response styles in all variables. As could be expected, their role is neglectable in the trace variables: as the obvious example of 'objective' data, these measures are not influenced by response styles. For example, ARS and DARS explain less than $1 \%$ of the variation of BlackBoard clicks. In contrast: there are variables for which response styles account for more than 
$25 \%$ of the variation at the other side of the spectrum. The different facets of anxiety are a great example. The Anxiety variable part of the MES instrument finds $26.2 \%$ of its variation explained by response styles, Anxious as a scale of epistemic emotions even more than one-third of explained variation and Learning Anxiety, scale of activity emotions, champions with $36.7 \%$ explained variation. However, the most remarkable observations in the left upper part of Table 2 refer to the course performance variables. Where one would have intuitively expected these variables to act as an objective measure, similar to the trace variables, they do no. For the three most crucial performance scores, the two examination scores and the final grade, explained variation by ARS and DARS is around $10 \%$, indicating that response styles have an undeniable role in predicting course performance.

Figure 2 provides a graphical representation of these data in the ARS versus DARS plane. Each dot represents a disposition variable, each square represents a course performance variable, and each diamond represents a trace variable. Where space allows, variable names are added. Such space is not available for the trace variables: they cluster around the origin, all carry relatively small ARS and DARS components. Course performance variables also cluster together, distant from the origin, having a relatively small, positive ARS component but a larger, positive DARS component. The only type of variable that occupies all quadrants of the ARS-DARS plane is the survey-based disposition type. We find two learning attitudes, Cognitive Competence and Affect, together with Academic Control, in the neighbourhood of the course performance variables, in the first quadrant: positive ARS and positive DARS components. All of these, together with Self-Belief, are self-efficacy type of variables. Only two variables occupy the second quadrant: negative ARS, positive DARS: NoDifficulty and Academic Buoyancy. We find many more variables, all of the maladaptive type, in the third quadrant: negative components of ARS and DARS. The epistemic and activity versions of Boredom, Hopelessness, and Disengagement are all examples. In the fourth quadrant, we find the above-mentioned group of anxiety-related variables and Confusion. These variables carry a positive ARS component and a negative DARS component. A large group of disposition variables is located at the right: large, positive ARS components, small, mostly positive DARS components.

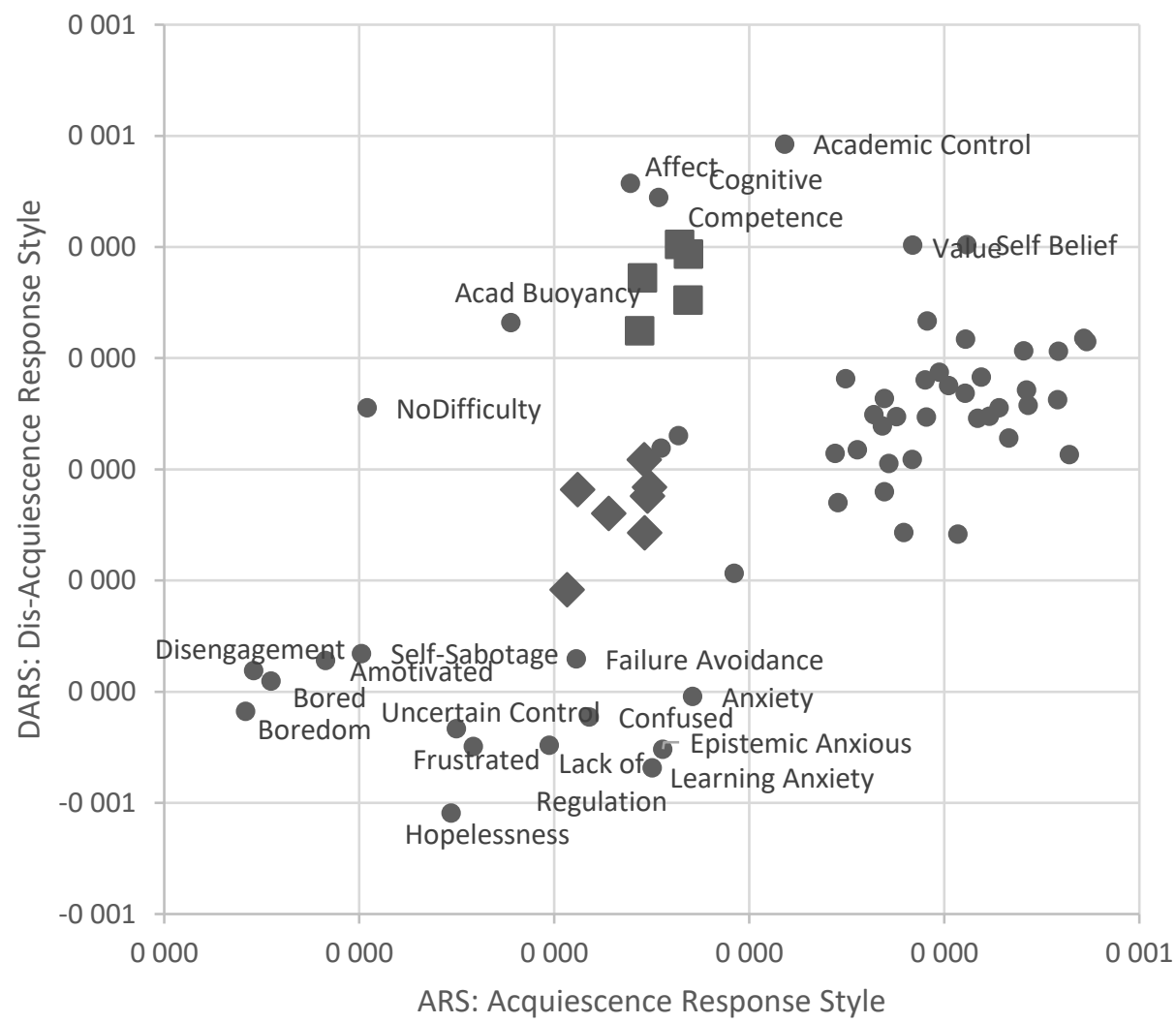

Figure 2. Regression betas of ARS and DARS from regressions of performance variables, denoted $\mathbf{\square}$, trace variables, denoted $\bullet$, and disposition variables, denoted $\bullet$, on ARS and DARS 


\section{DISCUSSION AND CONCLUSION}

The most appealing and successful learning analytics functionality is that of developing prediction models, signal students at risk of dropout or discover the optimal individual learning feedback. One can represent the task of predictive modeling with the help of the graphical representation provided above, realizing that the figure is limited to variation within the ARS-DARS dimensions: find a combination of trace and disposition variables that best approximates the course performance variable one wishes to predict. If we apply two predictors, this comes down to searching for two trace or disposition points in the graph that, once connected through a line, hit the point representing the performance variable to be predicted. Inspection of Figure 2 clarifies that the trace variables will never be part of such set of predictors: all situated around the origin of the ARS-DARS plane, they are not instrumental in predicting the response style component in the performance variables. Given the position of these performance variables, the preferred combination of predictors will be taken from the first quadrant, the adaptive, self-efficacy-based dispositions, and the third quadrant, the maladaptive dispositions.

A second conclusion refers to the consistency of the typology of related scales. Although the timing of the epistemic emotions instrument at the start of the course differs strongly from the activity emotions instrument halfway's timing, all three anxiety-related scales consistently position themselves in the same quadrant of the ARS-DARS plane. Making the bias coming with response styles predictable, thus accountable.

Given the response style component in performance variables, optimal prediction models take these ARS and DARS components into account. One can do this by isolating these components from the disposition data and adding them to the set of trace variables, but seeking parsimonious models, one better applies these dispositional variables themselves. In other words: optimal LA prediction models are composed predictor variables of trace and disposition types, where dispostions are best taken as a mixture of adaptive variables, such as self-belief, and maladaptive variables, such as boredom or disengagement.

\section{REFERENCES}

Bolt, DM, Lu, Y, \& Kim, JS 2014, 'Measurement and control of response styles using anchoring vignettes: A model-based approach', Psychological Methods, vol. 19, no. 4, pp. 528-541. DOI 10.1037/ met0000016.

Buckingham Shum, S \& Deakin Crick, R 2012, 'Learning Dispositions and Transferable Competencies: Pedagogy, Modelling and Learning Analytics', in S Buckingham Shum, D Gasevic, \& R Ferguson (eds.), Proceedings of the 2nd International Conference on Learning Analytics and Knowledge. ACM, New York, NY, USA.

Cheung, GW, \& Rensvold, RB 2000, 'Assessing extreme and acquiescence response sets in cross-cultural research using structural equations modeling'. Journal of Cross-Cultural Psychology, vol. 31, no. 2, pp. 187-212. DOI 10.1177/0022022100031002003.

Dawson, S, Joksimovic, S, Poquet, O \& Siemens, G 2019, 'Increasing the Impact of Learning Analytics', in Proceedings of the 9th International Conference on Learning Analytics \& Knowledge (LAK19), Association for Computing Machinery, New York, NY, USA, pp. 446-455. DOI 10.1145/3303772.3303784.

Henninger M, \& Meiser T 2020, 'Different approaches to modeling response styles in divide-by-total item response theory models (part 1): A model integration’, Psychological Methods, vol. 25, no. 5, pp. 560-576. DOI 10.1037/met0000249. PMID: 33017166.

Henninger M, \& Meiser T 2020, 'Different approaches to modeling response styles in divide-by-total item response theory models (part 2): Applications and novel extensions', Psychological Methods, vol. 25, no. 5, pp. 577-595, DOI 10.1037/met0000268. PMID: 33017167.

Ifenthaler, D, \& Yau, JYK 2020, 'Utilising learning analytics for study success: a systematic review', Educational Technology Research and Development, vol. 68, no. 4, pp. 1961-1990, DOI10.1007/s11423-020-09788-z.

Knight, S, Wise, A, \& Ochoa, X 2019, 'Fostering an impactful field of learning analytics', Journal of Learning Analytics, vol. 6, no. 3, pp. 1-4. DOI 10.18608/jla.2019.63.1.

SOLAR 2020, Webinar eight: What Do We Mean by Rigour in Learning Analytics? (https://www.solaresearch.org/community/webinars/). $\quad$ Recordings available at https://www.youtube.com/watch?v=RxGa6MvCX6Y\&feature=youtu.be.

Tempelaar, D, Rienties, B, \& Nguyen, Q 2020, 'Subjective data, objective data and the role of bias in predictive modelling: Lessons from a dispositional learning analytics application', PLOS ONE, vol. 15, no. 6: e0233977, DOI 10.1371/journal.pone.0233977.

Winne, PH 2020, 'Construct and consequential validity for learning analytics based on trace data', Computers in Human Behavior, vol. 112, no. 106457, DOI 10.1016/j.chb.2020.106457. 